Appl. Set-Valued Anal. Optim. 1 (2019), No. 1, pp. 19-28

Available online at http://asvao.biemdas.com

https://doi.org/10.23952/asvao.1.2019.1.02

\title{
SOME GENERAL FIXED POINT THEOREMS ON TOPOLOGICAL VECTOR SPACES
}

\author{
SEHIE PARK ${ }^{1,2}$ \\ ${ }^{1}$ The National Academy of Sciences, Republic of Korea, Seoul 06579, Korea \\ ${ }^{2}$ Department of Mathematical Sciences, Seoul National University, Seoul 08826, Korea
}

\begin{abstract}
In this paper, we introduce some of general fixed point theorems on topological vector spaces. The first one is a general theorem for the better admissible multimaps and the second one for generalized upper hemicontinuous (g.u.h.c.) convex-valued multimaps. The third is new fixed point theorems for half-continuous multimaps on locally convex topological vector spaces.

Keywords. Better admissible multimap; Convex space; Fixed point; Generalized upper hemicontinuous multimap; Locally convex topological vector space.
\end{abstract}

2010 Mathematics Subject Classification. 47H04, 47H10, 54H25, 55M20.

\section{INTRODUCTION}

Since the Brouwer fixed point theorem first appeared in 1910, several hundred generalizations or equivalent formulations have been found. One of the well-known equivalent formulations of the Brouwer theorem is the Kakutani fixed point theorem in 1941 for convex-valued upper semicontinuous (u.s.c.) multimaps. This initiated the study of fixed points of multimaps in the last seven decades. The Kakutani theorem and its numerous generalizations were applied to game theory, mathematical economics, differential equations, systems and control theory, coincidence theory, minimax theory, variational inequalities, convex analysis, equilibria theory, and others.

Moreover, the compactness, convexity, upper semicontinuity, selfmapness, and finite dimensionality related to the Kakutani theorem are all extended, and further, for the case of infinite dimension, it is known that the domain and range of the relevant multimap may have different topologies. This is why the Kakutani theorem has so many generalizations; see [16].

In our previous works, we unified, improved, and generalized a lot of fixed point theorems on Kakutani maps defined on convex subsets of topological vector spaces. One of the main fixed point theorems in [16] is concerned with convex-valued generalized upper hemicontinuous (g.u.h.c) maps whose domains and ranges may have different topologies. Then we add general fixed point theorems for g.u.h.c. multimaps defined on convex subsets of locally convex topological vector spaces under certain compactness and boundary conditions.

On the other hand, for compact multimaps in the 'better' admissible class $\mathfrak{B}$ defined on abstract convex spaces, we found that they have almost fixed point property whenever their ranges are Klee approximable

E-mail addresses: park35@ snu.ac.kr; sehiepark@gmail.com.

Received March 4, 2019; Accepted March 22, 2019.

(C)2019 Applied Set-Valued Analysis and Optimization 
into the domains. This enables us to generalize and unify many fixed point theorems for various types of multimaps appeared in topology and nonlinear analysis; see, [11, 12, 14, 15, 19, 20, 21, 24] and the references therein.

Recently, based on the new KKM theory, the author [22] reviewed several fixed point theorems which unify more than one hundred known generalizations of the Brouwer theorem related to t.v.s. or topological fixed point theory.

On the other hand, in 2008, Hering et al. [4] obtained a generalization of the Brouwer fixed point theorem for possibly discontinuous maps defined on polytopes in $\mathbb{R}^{n}$. Later, Bich [3] extended their work to maps defined on compact convex subset of $\mathbb{R}^{n}$. Moreover, in [2], he defined a new class of half-continuous multimaps which includes maps in [4] and obtained fixed point theorems for the new class. Furthermore, Termwuttipong and Kaewtem [25] showed that some results in [2] are also valid in locally convex Hausdorff topological vector spaces.

Our aim in this review is to introduce a part of $[22,23]$ with some supplements. In fact, we introduce some of the above-mentioned general fixed point theorems on topological vector spaces. The first one is a general theorem for the better admissible multimaps and the second one is a new version for g.u.h.c. convex-valued multimaps. The third one is new fixed point theorems for half-continuous multimaps on locally convex topological vector spaces due to Termwuttipong and Kaewtem [25]. Finally, We compare some of our previous results on g.u.h.c. maps with the new results of [25].

\section{BETTER ADMISSIBLE MAPS $\mathfrak{B}$}

A multimap $F: X \multimap Y$ is a function from a set $X$ into the set $2^{Y}$ of nonempty subsets of $Y$; that is, a function with the values $F(x) \subset Y$ for $x \in X$ and the fibers $F^{-}(y):=\{x \in X \mid y \in F(x)\}$ for $y \in Y$. We sometimes use the term map instead of multimap. For $A \subset X$, let

$$
F(A):=\bigcup\{F(x) \mid x \in A\} .
$$

For any $B \subset Y$, the (lower) inverse of $B$ under $F$ is defined by

$$
F^{-}(B):=\{x \in X \mid F(x) \cap B \neq \emptyset\} .
$$

For topological spaces $X$ and $Y$, a map $F: X \multimap Y$ is said to be closed if its graph

$$
\operatorname{Gr}(F):=\{(x, y) \mid y \in F(x), x \in X\}
$$

is closed in $X \times Y$, and compact if $F(X)$ is contained in a compact subset of $Y$.

A map $F: X \multimap Y$ is said to be upper semicontinuous (u.s.c.) if, for each closed $\operatorname{set} B \subset Y, F^{-}(B)$ is closed in $X$; lower semicontinuous (1.s.c.) if, for each open set $B \subset Y, F^{-}(B)$ is open in $X$; and continuous if it is u.s.c. and l.s.c.

If $F: X \multimap Y$ is u.s.c. with closed values and if $Y$ is regular, then $F$ is closed. The converse is true whenever $Y$ is compact.

Usually, a Kakutani map is a u.s.c. map with closed convex values.

A t.v.s. means a real Hausdorff topological vector space $E$. Let $E^{*}$ denote the topological dual of $E$.

A polytope $P$ in a t.v.s. $E$ is a homeomorphic image of a simplex.

For topological spaces $X$ and $Y$, we define the 'better' admissible class $\mathfrak{B}$ of maps from $X$ into $Y$ as follows [14, 15, 23]: 
$F \in \mathfrak{B}(X, Y) \Longleftrightarrow F: X \multimap Y$ is a map such that, for any natural $n \in \mathbb{N}$, any continuous function $\phi: \Delta_{n} \rightarrow X$, and any continuous function $p: F \phi\left(\Delta_{n}\right) \rightarrow \Delta_{n}$, the composition

$$
\Delta_{n} \stackrel{\phi}{\longrightarrow} \phi\left(\Delta_{n}\right) \subset X \stackrel{F}{\circ} F \phi\left(\Delta_{n}\right) \stackrel{p}{\longrightarrow} \Delta_{n}
$$

has a fixed point.

Example 2.1. Subclasses of the better admissible class $\mathfrak{B}$ are classes of continuous functions $\mathbb{C}$, the Kakutani maps $\mathbb{K}$ (with convex values and codomains are convex spaces), the Aronszajn maps $\mathbb{M}$ (with $R_{\delta}$ values), the acyclic maps $\mathbb{V}$ (with acyclic values), the Powers maps $\mathbb{V}_{c}$ (finite compositions of acyclic maps), the O'Neill maps $\mathbb{N}$ (continuous with values of one or $m$ acyclic components, where $m$ is fixed), the approachable maps $\mathbb{A}$ (whose domains and codomains are subsets of t.v.s.), admissible maps of Górniewicz (1976), $\sigma$-selectionable maps of Haddad and Lasry (1983), permissible maps of Dzedzej (1985), the Fan-Browder maps (codomains are convex sets), locally selectionable maps having convex values, the class $\mathbb{K}_{c}^{+}$of Lassonde (1991), the class $\mathbb{V}_{c}^{+}$of Park et al. (1994), and approximable maps of Ben-El-Mechaiekh and Idizk (1994), the admissible class $\mathfrak{A}_{c}^{\kappa}$ due to Park (1993). For the literature denoted by the form (year); see $[12,13,14,15]$ and the references therein.

In 1998, the author obtained the following result.

Theorem 2.1. [15] Let $E$ be a t.v.s. and $X$ an admissible (in the sense of Klee) convex subset of $E$. Then any compact closed map $F \in \mathfrak{B}(X, X)$ has a fixed point.

In [15], it was shown that Theorem 2.1 subsumes more than sixty known or possible particular cases and generalizes them in terms of the involving spaces and multimaps as well. Later, further examples of maps in the class $\mathfrak{B}$ were known.

It is not known yet whether the admissibility of $X$ can be eliminated in Theorem 2.1.

Theorem 2.1 can be generalized by switching the admissibility of domain of the map to the Klee approximability of its ranges as follows:

Let $X$ be a subset of a t.v.s. $E$ and $\mathscr{V}$ the neighborhood system of 0 in $E$. A nonempty subset $K$ of $X$ is said to be Klee approximable into $X$ if for any $V \in \mathscr{V}$, there exists a continuous function $h: K \rightarrow X$ such that $x-h(x) \in V$ for all $x \in K$ and $h(K)$ is contained in a polytope in $X$.

Example 2.2. We give some examples of Klee approximable sets:

(1) If a subset $X$ of $E$ is admissible (in the sense of Klee), then every compact subset $K$ of $X$ is Klee approximable into $E$.

(2) Any polytope in a subset $X$ of a t.v.s. is Klee approximable into $X$.

(3) Any compact subset $K$ of a convex subset $X$ in a locally convex t.v.s. is Klee approximable into $X$.

(4) Any compact subset $K$ of a convex and locally convex subset $X$ of a t.v.s. is Klee approximable into $X$.

(5) Any compact subset $K$ of an admissible convex subset $X$ of a t.v.s. is Klee approximable into $X$.

(6) Let $X$ be an almost convex dense subset of an admissible subset $Y$ of a t.v.s. $E$. Then every compact subset $K$ of $Y$ is Klee approximable into $X$.

Note that $(6) \Rightarrow(5) \Rightarrow(4) \Rightarrow(3)$.

In 2004, Theorem 2.1 was generalized as follows: 
Theorem 2.2. [18] Let $X$ be a subset of a t.v.s. $E$ and $F \in \mathfrak{B}(X, X)$ a compact closed multimap. If $F(X)$ is Klee approximable into $X$, then $F$ has a fixed point.

The following results with $\mathfrak{B}^{p}=\mathfrak{B}$ were obtained in 2007 .

Corollary 2.1. [19] Let $X$ be an almost convex admissible subset of a t.v.s. $E$ and $F \in \mathfrak{B}(X, X)$ a compact closed map. Then $F$ has a fixed point.

Corollary 2.2. [19] Let $X$ be an almost convex subset of a locally convex t.v.s. $E$ and $F \in \mathfrak{B}(X, X) a$ compact closed map. Then $F$ has a fixed point.

One of the most simple known example is that every compact continuous selfmap on an almost convex subset in a Euclidean space has a fixed point. This generalizes the Brouwer fixed point theorem.

Moreover, since the class $\mathfrak{B}(X, X)$ contains a large number of special types of maps, we can apply Theorem 2.2 to them. Since a Kakutani map belongs to $\mathfrak{B}$, Theorem 2.2 and Corollaries 2.1 and 2.2 generalize the Himmelberg theorem.

Example 2.3. In the following, we list more than sixty results in chronological order, from which we can deduce particular forms of Theorem 2.2. Some results are direct consequences of Theorem 2.2 and for some cases we can obviously obtain particular forms of Theorem 2.2 for a subclass of $\mathfrak{B}$ and a special type of t.v.s. For the literature denoted by the form (year) in this section; see $[15,16]$ and the references therein.

Poincaré (1883, 1884), Bohl (1904), Brouwer (1912), Alexander (1922), Birkhoff and Kellogg (1922), Schauder (1927), Schauder (1930), Tychonoff (1935), von Neumann (1937), Krein and Šmulian (1940), Miranda (1940), Kakutani (1941), Eilenberg and Montgomery (1946), Beagle (1950), Bohnenblust and Karlin (1950), Hukuhara (1950), Nagumo (1951), Fan (1952), Glicksberg (1952), O’Neill (1957), Schaefer (1959), Nikaido (1959), Singbal (1962), Fan (1964), Powers (1970, 1972), Himmelberg (1972), Rhee (1972), Fournier and Granas (1973), Sehgal and Morrison (1973), Hahn and Pötter (1974), Górniewicz (1976), Krauthausen (1976), Skordev (1976), Idzik (1978), Ha (1980), Hadžić (1980), Girolo (1981), Górniewicz and Granas (1981), Ben-El-Mechaiekh, Deguire, et Granas (1982), Haddad and Lasry (1983), Arino, Gautier, and Penot (1984), Dzedzej (1985), Simons (1986), McLinden (1989), Ben-El-Mechaiekh (1990), Lassonde (1990), Ben-El-Mechaiekh (1991), Ben-El-Mechaiek and Deguire (1991), Lassonde (1991), Shioji (1991), Park (1992), Ben-El-Mechaiek (1993), Park (1993), Park, Singh, and Watson (1994), Chang and Yen (1996), Chu (1996), Park (1996), Park and H. Kim (1996), Park (1997), Park (2000).

The following is known as the Zima-Hadžić type fixed point theorem.

Corollary 2.3. Let $X$ be a convex subset of a t.v.s. $E$ and $T: X \multimap X$ a compact u.s.c. multimap with nonempty closed convex values. Then $T$ has a fixed point $x_{0} \in T\left(x_{0}\right)$ if the following holds:

(Z) for each neighborhood $U$ of 0 in $E$, there exists a neighborhood $V$ of 0 in $E$ such that

$$
\operatorname{co}(V \cap(T(X)-T(X))) \subset U .
$$

Corollary 2.4. [5] Let $X$ be a convex subset of a locally convex Hausdorff t.v.s. E and $T: X \multimap X a$ compact u.s.c. multimap with nonempty closed convex values. Then $T$ has a fixed point $x_{0} \in T\left(x_{0}\right)$. 
Recall that the Himmelberg theorem in 1972 unifies and generalizes historically well-known fixed point theorems due to Brouwer, Schauder, Tychonoff, Kakutani, Bohnenblust and Karlin, Fan, Glicksberg, Hukuhara, Rhee, and others.

More early in 1941, in order to give simple proofs of von Neumann's intersection lemma and his minimax theorem, Kakutani [6] obtained the following generalization of the Brouwer theorem to multimaps.

Corollary 2.5. [6] If $x \mapsto \Phi(x)$ is an upper semicontinuous point-to-set mapping of an r-dimensional closed simplex $S$ into the family of closed convex subsets of $S$, then there exists an $x_{0} \in S$ such that $x_{0} \in \Phi\left(x_{0}\right)$.

It is equivalent to the following.

Corollary 2.6. [6] Corollary 2.5 is also valid even if $S$ is an arbitrary bounded closed convex set in an Euclidean space.

\begin{tabular}{|c|ll|ll|}
\hline \hline \multirow{2}{*}{$E$} & \multicolumn{2}{|c|}{$f: K \longrightarrow K$} & & \multicolumn{2}{c|}{$F: K \multimap K$} \\
\hline I & Brouwer & 1912 & Kakutani & 1941 \\
\hline II & Schauder & 1927,1930 & $\begin{array}{l}\text { Bohnenblust } \\
\text { and Karlin }\end{array}$ & 1950 \\
\hline III & Tychonoff & 1935 & Fan & 1952 \\
& Hukuhara & 1950 & Glicksberg & 1952 \\
\hline IV & Fan & 1964 & Himmelberg & 1972 \\
& & & Granas and Liu & 1988 \\
\hline V & Zima & 1977 & Hadžić & $1981,1982,1987$ \\
& Rzepecki & 1979 & & \\
\hline
\end{tabular}

The major particular forms of Corollary 2.3 for Kakutani maps $F: K \multimap K$, where $K$ is a nonempty compact convex subset, can be adequately summarized by the preceding diagram. In the diagram, $f$ denotes a (single-valued) continuous function and $F$ a Kakutani map. $K$ in the class $I$ is a compact convex subset of Euclidean spaces, II normed vector spaces, III locally convex t.v.s., and IV t.v.s. $E$ on which $E^{*}$ separates points. Moreover, $K$ in the class $\mathrm{V}$ is a convex subset of a t.v.s. such that $f(K)$ and $F(K)$ are relatively compact subsets of the Zima type.

Finally, in this section, note that O'Regan and Perán [9] set out a rigorous presentation of Park's class of admissible multimaps, within the general framework of multimaps between topological spaces, using a broad definition of convexity. In addition, they obtain a fixed point theorem for better admissible multimaps defined on a proximity space via the Samuel-Smirnov compactification.

\section{GENERALIZED UPPER HEMICONTINUOUS MULTIMAPS}

The class of u.s.c. multimaps on t.v.s. is further extended as follows: For a topological space $X$ and a t.v.s. $E$, a map $F: X \multimap E$ is said to be

(i) upper demicontinuous (u.d.c.) if for each $x \in X$ and open half-space $H$ in $E$ containing $F(x)$, there exists an open neighborhood $N$ of $x$ in $X$ such that $F(N) \subset H$; 
(ii) upper hemicontinuous (u.h.c.) if for each $h \in E^{*}$ and for any real $\alpha$, the set $\{x \in X \mid \sup h F(x)<$ $\alpha$ \} is open in $X$; and

(iii) generalized u.h.c. if for each $p \in E^{*}$, the set $\{x \in X \mid \sup p F(x) \geq p(x)\}$ is closed in $X$.

In our earlier works $[11,17]$, the author unified a large number of generalizations of the Kakutani theorem to maps of the above-mentioned types.

According to Lassonde [7], a convex space $X$ is a nonempty convex set with any topology that induces the Euclidean topology on the convex hulls of its finite subsets. A nonempty subset $L$ of a convex space $X$ is called a $c$-compact set if for each finite set $N \subset X$ there is a compact convex set $L_{N} \subset X$ such that $L \cup N \subset L_{N}$.

Let $c c(E)$ denote the set of nonempty closed convex subsets of a t.v.s. $E$ and $k c(E)$ the set of nonempty compact convex subsets of $E$.

Let $X$ be a nonempty convex subset of a vector space $E$. The algebraic boundary $\delta_{E}(X)$ of $X$ in $E$ is the set of all $x \in X$ for which there exists $y \in E$ such that $x+r y \notin X$ for all $r>0$. If $E$ is a t.v.s., the topological boundary $\mathrm{Bd} X=\operatorname{Bd}_{E} X$ of $X$ is the complement of $\operatorname{Int}_{E} X$ in the closure $\bar{X}$. It is known that $\delta_{E}(X) \subset \mathrm{Bd} X$ and in general $\delta_{E}(X) \neq \mathrm{Bd} X$.

Let $X \subset E$ and $x \in E$. The inward and outward sets of $X$ at $x, I_{X}(x)$ and $O_{X}(x)$, resp., are defined as follows

$$
I_{X}(x):=x+\bigcup_{r>0} r(X-x), \quad O_{X}(x):=x+\bigcup_{r<0} r(X-x) .
$$

For $p \in E^{*}$ and $U, V \subset E$, let

$$
d_{p}(U, V)=\inf \{|p(u-v)|: u \in U, v \in V\} .
$$

The following is a two map version of the main theorem in $[11,17]$.

Theorem 3.1. Let $X$ be a convex space, $L$ a c-compact subset of $X, K$ a nonempty compact subset of $X$, $E$ a t.v.s. containing $X$ as a subset, and $F, G: X \multimap E$ maps maps satisfying $F \subset G$ and either

(A) $E^{*}$ separates points of $E$ and $G: X \rightarrow k c(E)$, or

(B) $E$ is locally convex and $G: X \rightarrow c c(E)$.

(I) Suppose that, for each $p \in E^{*}$,

(0) $\left.p\right|_{X}$ is continuous on $X$;

(1) $X_{p}:=\{x \in X \mid \inf p(F(x)) \leq p(x)\}$ is closed in $X$;

(2) $d_{p}\left(F(x), \overline{I_{X}(x)}\right)=0$ for every $x \in K \cap \delta_{E}(X)$; and

(3) $d_{p}\left(F(x), \overline{I_{L}(x)}\right)=0$ for every $x \in X \backslash K$.

Then there exists an $x \in X$ such that $x \in G(x)$.

(II) Suppose that, for each $p \in E^{*}$,

$\left.(0)^{\prime} p\right|_{X}$ is continuous on $X$;

$(1)^{\prime} X_{p}:=\{x \in X \mid \sup p(F(x)) \geq p(x)\}$ is closed in $X$;

(2) $^{\prime} d_{p}\left(F(x), \overline{O_{X}(x)}\right)=0$ for every $x \in K \cap \delta_{E}(X)$; and

(3) ${ }^{\prime} d_{p}\left(F(x), \overline{O_{L}(x)}\right)=0$ for every $x \in X \backslash K$.

Then there exists an $x \in X$ such that $x \in G(x)$. Further, if $F=G$ is u.h.c., then $F(X) \supset X$.

The major particular forms of Theorem 3.1 can be adequately summarized by the following enlarged version of the diagrams previously given in $[11,16]$. 
In the diagram, the class I stands for that of Euclidean spaces, II for normed vector spaces, III for locally convex t.v.s., and IV for t.v.s. having sufficiently many linear functionals. Moreover, $f$ stands for single-valued maps and $F$ for set-valued maps; and $K$ stands for a nonempty compact convex subset of a space $E$, and $X$ for a nonempty convex subset of $E$ satisfying certain coercivity conditions with respect to $F: X \multimap E$ with certain boundary conditions.

\begin{tabular}{|c|c|c|c|c|}
\hline E & \multicolumn{2}{|c|}{$f: K \longrightarrow K$} & \multicolumn{2}{|c|}{$F: K \multimap K$} \\
\hline I & Brouwer & 1912 & Kakutani & 1941 \\
\hline II & Schauder & 1927,1930 & $\begin{array}{l}\text { Bohnenblust } \\
\text { and Karlin }\end{array}$ & 1950 \\
\hline III & Tychonoff & 1935 & $\begin{array}{l}\text { Fan } \\
\text { Glicksberg }\end{array}$ & $\begin{array}{l}1952 \\
1952\end{array}$ \\
\hline IV & Fan & 1964 & Granas and Liu & 1986 \\
\hline & \multicolumn{2}{|c|}{$f: K \longrightarrow E$} & \multicolumn{2}{|c|}{$F: K \multimap E$} \\
\hline I & $\begin{array}{l}\text { Bohl } \\
\text { Knaster, Kuratowski } \\
\text { and Mazurkiewicz }\end{array}$ & $\begin{array}{l}1904 \\
1929\end{array}$ & & \\
\hline II & Rothe & 1938 & & \\
\hline III & $\begin{array}{l}\text { Halpern } \\
\text { Fan } \\
\text { Reich } \\
\text { Sehgal and Singh }\end{array}$ & $\begin{array}{l}1965 \\
1969 \\
1972 \\
1983\end{array}$ & $\begin{array}{l}\text { Browder } \\
\text { Fan } \\
\text { Glebov } \\
\text { Halpern } \\
\text { Cellina } \\
\text { Reich } \\
\text { Cornet } \\
\text { Lasry and Robert } \\
\text { Simons }\end{array}$ & $\begin{array}{l}1968 \\
1969 \\
1969 \\
1970 \\
1970 \\
1972,1978 \\
1975 \\
1975 \\
1986\end{array}$ \\
\hline IV & $\begin{array}{l}\text { Halpern and Bergman } \\
\text { Kaczynski } \\
\text { Roux and Singh } \\
\text { Sehgal, Singh } \\
\text { and Whitfield }\end{array}$ & $\begin{array}{l}1968 \\
1983 \\
1989 \\
1990\end{array}$ & $\begin{array}{l}\text { Granas and Liu } \\
\text { Park } \\
\text { Balaj }\end{array}$ & $\begin{array}{l}1986 \\
1988,1991 \\
2002\end{array}$ \\
\hline & & & $F: X$ & \\
\hline II & & & Ding and Tan & 1992 \\
\hline III & & & $\begin{array}{l}\text { Fan } \\
\text { Shih and Tan } \\
\text { Jiang }\end{array}$ & $\begin{array}{l}1984 \\
1987,1988 \\
1988\end{array}$ \\
\hline IV & & & $\begin{array}{l}\text { Park } \\
\text { Yuan, Smith, and Lou }\end{array}$ & $\begin{array}{l}1992,1993 \\
1998\end{array}$ \\
\hline
\end{tabular}

In fact, Theorem 3.1 implies all of the fixed point theorems in the diagram. Note that, in the diagram, Bohl's theorem in 1904 was well-known to be equivalent to the Brouwer fixed point theorem in 1912.

Remark 3.1. Theorem 3.1 is a two map version of the main theorem in $[11,17]$ for $F=G$. This is motivated by Balaj [1].

From Theorem 3.1, we obtain the following result.

Corollary 3.1. Let $K$ be a nonempty compact convex subset of a t.v.s. E on which $E^{*}$ separates points and let $F, G: K \multimap E$ be two maps such that 

(a) $F \subset G$;
(b) $F$ is g.u.h.c.;
(c) G has closed convex values;
(d) $F(y) \cap \overline{I_{K}(y)} \neq \emptyset$ [resp., $F(y) \cap \overline{O_{K}(y)} \neq \emptyset$ ] for each $y \in K$.

Then $G$ has a fixed point $y_{0} \in K$.

Balaj's unification [1, Theorem 3] of Halpern's theorems is a particular form of Corollary 3.1 where $F$ is u.h.c. and $E$ is a locally convex t.v.s.

Corollary 3.2. Let $K$ be a nonempty compact convex subset of a t.v.s. E on which $E^{*}$ separates points and $F: K \multimap K$ a g.u.h.c. map. Then $G:=\overline{\mathrm{co}} F: K \multimap K$ has a fixed point.

In case $F=G$, corollary 3.2 reduces to the following.

Corollary 3.3. Let $K$ be a nonempty compact convex subset of a t.v.s. $E$ on which $E^{*}$ separates points and $F: K \rightarrow k c(K)$ a g.u.h.c. map. Then $F$ has a fixed point.

\section{NEW RESULTS ON HALF-CONTINUOUS MULTIMAPS}

In this section, we follow [25].

Definition 4.1. [2] Let $C$ be a subset of a t.v.s. $E$. A map $f: C \rightarrow E$ is said to be half-continuous if for each $x \in C$ with $x \neq f(x)$ there exists $p \in E^{*}$ and a neighborhood $W$ of $x$ in $C$ such that

$$
\langle p, f(y)-y\rangle>0
$$

for all $y \in W$ with $y \neq f(y)$.

Theorem 4.1. [25] Let $C$ be a nonempty compact convex subset of a locally convex t.v.s. E. If $f: C \rightarrow C$ is half-continuous, then $f$ has a fixed point.

It is noted in [25] that this theorem generalizes theorems due to Brouwer, Schauder, and Tychonoff.

Definition 4.2. [25] Let $C$ be a subset of a t.v.s. $E$. A multimap $F: C \multimap E$ is said to be half-continuous if for each $x \in C$ with $x \notin F(x)$ there exists $p \in E^{*}$ and a neighborhood $W$ of $x$ in $C$ such that

$$
\forall y \in W, y \notin F(y) \Longrightarrow \forall z \in F(y),\langle p, z-y\rangle>0 .
$$

Lemma 4.1. [25] Let $C$ be a nonempty subset of a t.v.s. $E$ and $F: C \multimap C$. If $F$ is half-continuous, then $F$ has a half-continuous selection $f: C \rightarrow E$ such that $x=f(x)$ if $x \in F(x)$.

Theorem 4.2. [25] Let $C$ be a nonempty compact convex subset of a locally convex t.v.s. E. If $F: C \multimap C$ is half-continuous, then $F$ has a fixed point.

It is noted in [25] that this theorem generalizes the case $F$ is upper demicontinuous with closed convex values and theorems due to Kakutani, Fan, and Glicksberg.

For a nonself map $f: C \rightarrow E$, we have the following:

Theorem 4.3. [25] Let $C$ be a nonempty compact convex subset of a locally convex t.v.s. E. Suppose that $F: C \rightarrow E$ is half-continuous and for each $x \in C$ with $x \neq f(x)$ there exists $\lambda<1$ such that $\lambda x+(1-$ $\lambda) f(x) \in C$. Then $f$ has a fixed point.

The following concept originates from Halpern:

Definition 4.3. Let $C$ be a subset of a vector space $E$. A multimap $F: C \multimap E$ is said to be inward [resp., outward] if for each $x \in C$ there exists $y \in F(x)$ and $\lambda>0$ [resp., $\lambda<0$ ] satisfying $x+\lambda(y-x) \in C$.

Theorem 4.4. [25] Let $C$ be a nonempty compact convex subset of a locally convex t.v.s. E. Then every inward (or outward) half-continuous multimap $F: C \multimap E$ has a fixed point. 
Theorems 4-7 in [25] are all for inward (or outward) maps defined on compact convex sets.

Problem. Are there any generalizations of Theorems 4-7 in [25] relaxing compactness or boundary conditions as in Section 3?

\section{COMPARISONS TO OUR PREVIOUS RESULTS}

Lemma 5.1. Let $C$ be a nonempty subset of a locally convex t.v.s. E. If $F: C \multimap E$ is a generalized u.h.c. map with nonempty closed convex values, then $F$ is half continuous.

Proof. Let $x \in C$ such that $x \notin F(x)$. Since $E$ is locally convex and $F(x)$ is closed convex, by the standard separation theorem, there exists $p \in E^{*}$ such that inf $p F(x)>p(x)$. Let

$$
W_{x}:=\{y \in C \mid \inf p F(y)>p(y)\},
$$

which is open since $F$ is generalized u.h.c. Note that $x \in W_{x} \subset C$. Therefore

$$
\forall y \in W_{x}, y \notin F(y) \Longrightarrow \forall z \in F(y),\langle p, z-y\rangle>0 .
$$

This shows $F$ is half-continuous.

Another standard separation theorem also works for the following:

Lemma 5.2. Let $C$ be a nonempty subset of a t.v.s. $E$ on which $E^{*}$ separates points. If $F: C \multimap E$ is a g.u.h.c. map with nonempty compact convex values, then $F$ is half continuous.

Lemmas 5.1 and 5.2 reduce to [25, Propositions 4.2 and 4.4], resp., when $F$ is u.d.c. At present, we do not have any examples which are not satisfying the converses of Lemmas 5.1 and 5.2.

In 1991, we obtained the following:

Theorem 5.1. [10] Let $X$ be a nonempty compact convex subset of a t.v.s. E on which $E^{*}$ separates points, and $f: X \rightarrow E$ a weakly inward [resp., outward] function such that

$$
\{x \in X \mid p(x)<p(f(x))\}
$$

is open for all $p \in E^{*}$. Then $f$ has a fixed point.

Note that $f$ is half-continuous by Lemma 5.1 and that Theorem 5.1 is not comparable to Theorem 4.4.

The following forms of Corollaries 3.2 and 3.3 follow from results of [25]:

Theorem 5.2. Let $K$ be a nonempty compact convex subset of a locally convex t.v.s. E and $F: K \multimap K a$ g.u.h.c. map. Then $G:=\overline{\mathrm{co}} F: K \multimap K$ has a fixed point.

In fact, $G$ is easily seen to be g.u.h.c. and hence half-continuous by Lemma 5.2. Therefore, Theorem 5.2 follows from Theorem 4.2.

The following is a 2019 version of the Fan-Glicksberg theorem; see [16].

Corollary 5.1. Let $K$ be a nonempty compact convex subset of a locally convex t.v.s. E and $F: K \rightarrow k c(K)$ a g.u.h.c. map. Then $F$ has a fixed point.

\section{REFERENCES}

[1] M. Balaj, A unified generalization of two Halpern's fixed point theorems and applications, Numer. Funct. Anal. Optim. 23 (2002), 105-111

[2] P. Bich, Some fixed point theorems for discontinuous mappings, Cahiers de la Maison des Sciences Economiques, b06066 (2006), 1 C10.

[3] P. Bich, t An answer to a question by Hering et al. Oper. Res. Lett. 36 (2008), 525-526.

[4] P.J.-J. Herings, G. van der Laan, D. Talman, Z. Yang, A fixed point theorem for discontinuous functions, Oper. Res. Lett. 36 (2008), 89-93.

[5] C.J. Himmelberg, Fixed points of compact multifunctions, J. Math. Anal. Appl. 38 (1972), 205-207. 
[6] S. Kakutani, A generalization of Brouwer's fixed-point theorem, Duke Math. J. 8 (1941), 457-459.

[7] M. Lassonde, On the use of KKM multifunctions in fixed point theory and related topics, J. Math. Anal. Appl. 97 (1983), 151-201.

[8] D. O'Regan, R.P. Agarwal, Fixed point theory for admissible multimaps defined on closed subsets of Fréchet spaces, J. Math. Anal. Appl. 277 (2003), 438-445.

[9] D. O’Regan, J. Perán, Fixed points for better admissible multifunctions on proximity spaces, J. Math. Anal. Appl. 380 (2011), 882-887.

[10] S. Park, A generalization of the Brouwer fixed point theorem, Bull. Korean Math. Soc. 28 (1991), 33-37.

[11] S. Park, Fixed point theory of multifunctions in topological vector spaces, J. Korean Math. Soc. 29 (1992), 191-208.

[12] S. Park, Fixed point theory of multifunctions in topological vector spaces, II, J. Korean Math. Soc. 30 (1993), 413-431.

[13] S. Park, Foundations of the KKM theory via coincidences of composites of upper semicontinuous maps, J. Korean Math. Soc. 31 (1994), 493-519

[14] S. Park, Coincidence theorems for the better admissible multimaps and their applications, Nonlinear Anal. 30 (1997) 4183-4191.

[15] S. Park, A unified fixed point theory of multimaps on topological vector spaces, J. Korean Math. Soc. 35 (1998), 803-829.

[16] S. Park, Ninety years of the Brouwer fixed point theorem, Vietnam J. Math. 27 (1999), 193-232.

[17] S. Park, Fixed points of generalized upper hemicontinuous maps, revisited, Acta Math. Vietnam. 27 (2002), 141-150.

[18] S. Park, Fixed points of multimaps in the better admissible class, J. Nonlinear Convex Anal. 5 (2004), 369-377.

[19] S. Park, Fixed point theorems for better admissible multimaps on almost convex sets, J. Math. Anal. Appl. 329 (2007), 690-702.

[20] S. Park, A unified fixed point theory in generalized convex spaces, Acta Math. Sinica, Eng. Ser. 23 (2007) $1509-1536$.

[21] S. Park, Fixed point theory of multimaps in abstract convex uniform spaces, Nonlinear Anal. 71 (2009) 2468-2480.

[22] S. Park, Applications of the KKM theory to fixed point theory, J. Nat. Acad. Sci. ROK, Nat. Sci. Ser. 50 (2011), 1-49.

[23] S. Park, Applications of multimap classes in abstract convex spaces, J. Nat. Acad. Sci. ROK, Nat. Sci. Ser. 51 (2012), $1-27$.

[24] S. Park, Corrections to "A unified fixed point theory of multimaps on topological vector spaces", J. Korean Math. Soc. 36 (1999), 829-832.

[25] I. Termwuttipong, T. Kaewtem, Fixed point theorems for half-continuous mappings on topological vector spaces, Fixed Point Theory Appl. 2010 (2010), Article ID 814970. 